\title{
An fMRI Compatible Touchscreen to Measure Hand Kinematics during a Complex Drawing Task
}

\author{
L. Braadbaart ${ }^{1,2}$, G. Buchan ${ }^{1}$, J. H. G. Williams ${ }^{2,3}$ and G. D. Waiter ${ }^{1,2^{\star}}$ \\ ${ }^{1}$ Aberdeen Biomedical Imaging Centre, University of Aberdeen, Lilian Sutton Building, \\ Aberdeen AB25 2ZD, UK \\ ${ }^{2}$ SINAPSE Collaboration, UK. \\ ${ }^{3}$ Clinical Research Centre, Division of Applied Health Sciences, University of Aberdeen, \\ Royal Cornhill Hospital, Aberdeen AB25 2ZH, UK.
}

\section{Authors' contributions}

This work was carried out in collaboration with all authors. Authors GDW, LB and JHGW designed the study. Authors $L B$ and GB performed the data collection, author $L B$ and GDW managed the analyses

of the study, author GDW wrote the first draft of the manuscript. All authors read and approved the final manuscript.

Article Information

DOI: 10.9734/BJAST/2015/17411 Editor(s):

(1) Lesley Diack, School of Pharmacy and Life Sciences, Robert Gordon University, UK. Reviewers:

(1) Or Cohen-Inbar, Department of Neurological Surgery, University of Virginia Health Care Center, Virginia.

(2) Tiago Atalaia, Portuguese Red Cross Health School, Portugal. Complete Peer review History: http://www. sciencedomain.org/review-history.php?iid=1139\&id=5\&aid=9422

ABSTRACT

Introduction: The measurement of human motion, or kinematics, is becoming increasingly important. More specifically the measurement of human motor control can give important information about a number of cognitive processes. However, due to the restricted environment of functional magnetic resonance imaging most studies involving kinematics have involved simple hand movements. We used a resistive touchscreen to measure the kinematics of human fine-motor control while simultaneously determining the neural basis of that control using functional magnetic resonance imaging (fMRI).

Methods: Eighteen participants aged 21 to 45 years (10 female) were recruited. During a 10 minute functional imaging session we recorded both the functional and kinematic response to a dynamic drawing task. We determined the impact of the active touchscreen in the MR environment by determining measures of signal drift and fluctuation. Maps of average neuronal response to the dynamic drawing task as well as measures of drawing ability were determined. 
Results: We found no apparent effect of the touchscreen on signal either signal drift or fluctuation. We found significant functional activation in bilateral cerebellum, as well as the insula, and left supramarginal, postcentral and precentral gyri, consistent with previous findings.

Conclusion: In conclusion we have shown the neural responses to a dynamic drawing task performed with a resistive touchscreen within an MRI scanner are reliable and consistent with previous work and that kinematic information can reliably reproduce drawing shape.

Keywords: fMRl; motor control; learning; kinematics.

\section{INTRODUCTION}

The measurement of human motion, or kinematics, is becoming increasingly important. More specifically the measurement of human motor control can give important information about a number of cognitive processes [1,2]. Due to the restricted environment of functional Magnetic Resonance Imaging (fMRI), most studies investigating kinematics have used simple hand movements (e.g. $[3,4]$ ) or the mere observation of movements (e.g. $[5,6]$ ) to study the neural correlates of motor control. These studies do not allow for the quantification of kinematics, and therefore are unable to look at individual differences in motor control and other kinematic domains. A previous study by Braadbaart et al. [7] has compared neural activation on a simple handle-moving task to quantified performance on a manual imitation drawing task completed outside the scanner, and found a relationship between individual differences in ability and neural activation patterns. To be able to take this research further, and look at neural activations during a complex task that records kinematics, an MRI-compatible drawing device had to be developed. Both Reitz et al. [8] and Tam et al. [9] have demonstrated the feasibility of using computer-based pads for the study of drawing during fMRI. Reitz et al. used an LED based optical system and colour gradient to detect position while Tam et al. employed a resistive touchscreen similar to that on automated bank cash-machines. Gold, Pomplun, Rice \& Sekuler [10] have previously used a fingerless glove to record kinematics by quantifying finger movements. They tested their method by tracking improvement over time. We aimed to implement a resistive touchscreen in an MRI environment by using locally sourced parts while reducing the potential for unwanted interference by removing the need for direct electrical connection between the touchscreen and the controlling computer. We therefore present an MRI compatible touchscreen system that is capable of precisely measuring the kinematics of hand movement that is optically isolated from the controlling PC outside the scan room. We tested the efficacy of this set-up firstly by first seeing if there was any interference between the scanner and the touchscreen, secondly by using a novel shape error method and seeing if participants improved on the task over time, and thirdly by seeing how areas of activation match up to those found in previous studies.

\section{METHODS}

\subsection{Touchscreen}

The touchscreen assembly, Fig. 1, was built from a resistive touchscreen, housed in an acrylic chassis, connected via a USB controller and a USB to fibre optic converter to a standard PC. The touchscreen was a $270 \mathrm{~mm}$ diagonal (active area $215 \mathrm{~mm} \times 164 \mathrm{~mm}$ ) 5-wire analogue resistive system with Indium Tin Oxide (ITO) conducting surfaces (Higgstec, Model Number T104S-5RA003N-0A18R0-200FH, Taiwan) with a matching USB controller (Higgstec, Model Number -USB-R5W-HT-580-R, Taiwan), which was USB1.1 full speed HID 1.11 compliant with a temporal resolution of 86 points per second, and a maximum response time of $20 \mathrm{~ms}$. The touchscreen, situated in the scan room, was connected to the PC via a fibre optic USB extender (USB Rover 200, Icron Technologies). Fiber optic transmission provides good reliability and immunity to interference including complete electrical isolation between the $P C$ and the touchscreen, essential in an MRI environment. The touchscreen was powered from the scan room isolated power supply, further reducing the possibility of interference.

\subsection{Participants}

Eighteen participants ( 10 female) between 21 and 45 years old were recruited, with a mean age of 26.4 (SD: 6.43 median: 24.3; range: 21.3-45.7) 


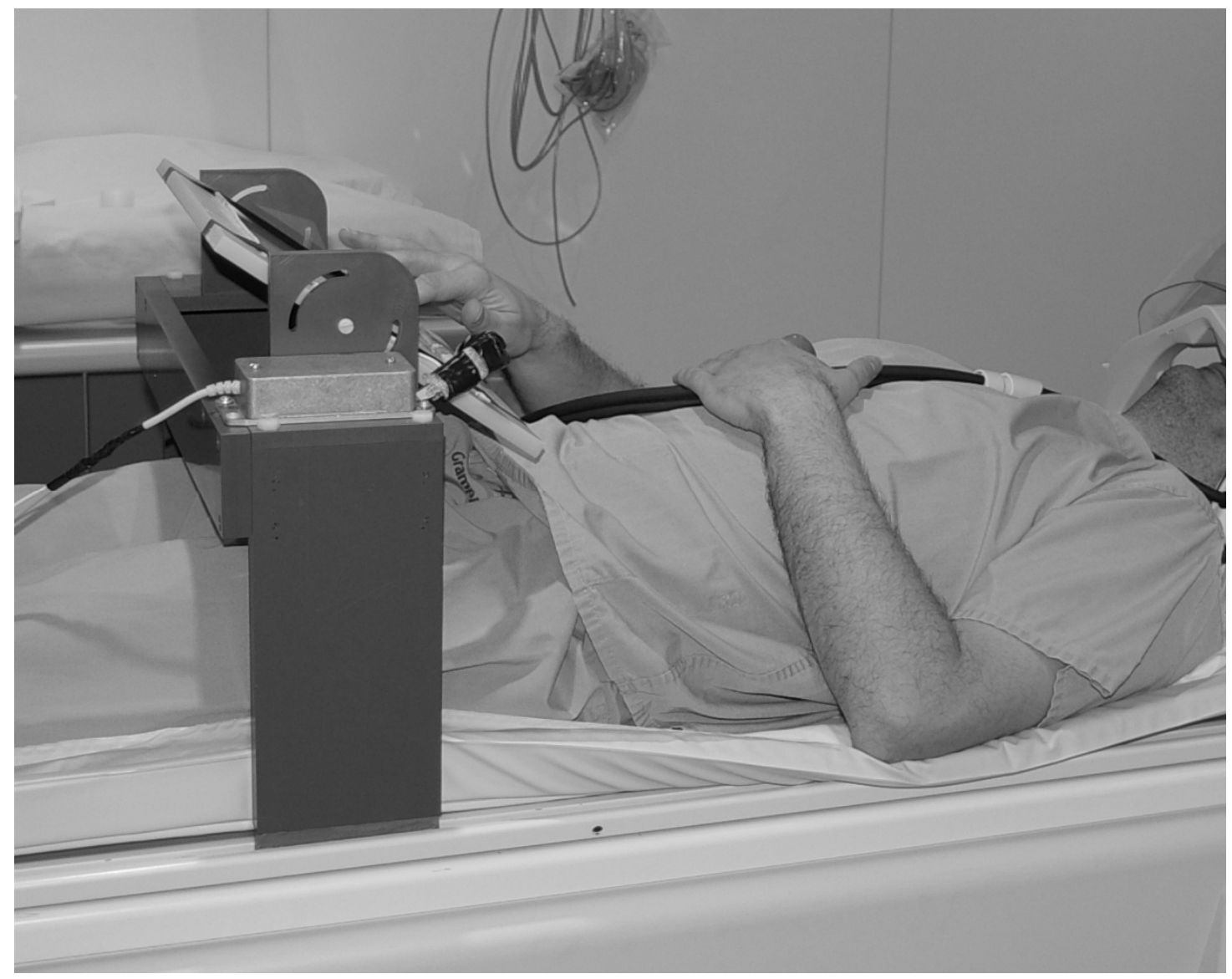

Fig. 1. Touchscreen in-situ showing acrylic chassis and tilting mechanism

years. All participants were right-handed, and were asked to confirm an absence of psychiatric disorder or history of illnesses that could affect the brain at time of consent. The study was approved by the College Ethics Review Board of the University of Aberdeen and all participants provided written, informed consent.

\subsection{Imaging}

MRI data was collected using a 3.0 T scanner (Achieva X-series, Philips Medical, Best, The Netherlands). A 32-channel phased-array head coil was used to obtain high-resolution gradient echo 3D volumetric images and a set of functional images using BOLD contrast. The high-resolution images were collected using the following parameters: field of view, $24 \mathrm{~cm}$; TR/TE 8.2/3.8 ms; flip angle, $8^{\circ}$; slices, 160; slice thickness, $1.0 \mathrm{~mm}$; matrix, $240 \times 240$. Functional MR images were acquired in the axial plane with a T2*-weighted single shot, gradient-echo, echoplanar pulse sequence with the following parameters: FOV, $24 \mathrm{~cm}$; 2000/30, TR/TE; flip angle, $78^{\circ}$, slices, 30; slice thickness, $5 \mathrm{~mm}$; matrix, 96x96. The head was firmly stabilized in the head coil with headphones and foam pads.

\subsection{Drawing task}

The drawing task used to test the touchscreen was a dynamic drawing task in response to a model produced action. Participants were presented in the scanner with videos and the output of their touchscreen interactions using Presentation (version 14, www.neurobs.com). There were two types dynamic drawing actions presented as videos; either the participants saw a model drawing a shape with their finger using the same touchscreen they had with them in the scanner (Draw), or they saw the same model with a dot moving across the screen that drew out the same shapes the model had been drawing (Observe). After each video, a screen came up with start and finish boxes on a white background; the participants used this to see the 
shape they were drawing, without having to look down at the touchscreen. The next video started as soon as the participant had reached the finish box. One example of each type of video was used first as a practice without scanning, to get participants comfortable drawing with their finger. The task was run twice, taking approximately 10.5 minutes each (310 volumes), depending on the speed at which the participants drew.

\section{5 fMRI Analysis}

Functional MRI data was analysed using MATLAB software with SPM8 (http://www.fil.ion.ucl.ac.uk/spm/software/spm8/). The functional images for each run were realigned to the first image of the run (maximum acceptable translation $5 \mathrm{~mm}$ and rotation 5 degrees in all directions). The mean scan from realignment was co-registered to the $\mathrm{T} 1$, and the T1 segmented. Scans were then normalized to the T1 grey matter image using the standard SPM MNI template and smoothed with an $8 \mathrm{~mm}$ FWHM Gaussian kernel. The smoothed images were modelled using a General Linear Model. The onset of each stimulus was assigned to either Draw or Observe, according to the relevant video, to generate t-tests. A standard high-pass filter was employed with 128s cut-off, using the movement data from realignment as a regressor of no interest.

To make inferences at the group level activation patterns from the individual t-tests were averaged across the 18 participants. This revealed the difference in activation between Draw and Observe. Group results employed a voxel threshold of $p<.05$, FWE-corrected. The Talairach atlas and AAL toolbox were used to define anatomical regions.

\subsection{Image Quality Assessment}

Image quality was assessed by following the guidelines given by Friedman and Glover [11]. To provide a realistic assessment of the possible impact of the touchscreen on image quality, the signal from a region of interest placed in the thalamus of a single participant was used. Data was collected during 3 separate runs where 310 volumes were collected in each. The first was with the participant performing a typical block designed drawing task with visual cues, the second was with the touchscreen in place and the participant viewing the visual cues but not performing any movement and the third was after the touchscreen had been removed from the scan room. For comparison a fourth set of data was collected with a standard mineral oil phantom. Signal to fluctuation noise ratio (SFNR) was calculated by dividing the ROI time series mean by its standard deviation once the series had been de-trended by a $2^{\text {nd }}$ degree polynomial. Drift was determined by subtracting the maximum trend signal intensity from the minimum trend signal. Percentage fluctuation was determined by dividing ROI time series standard deviation by its mean and multiplying by 100.

\subsection{Reproducibility of Figure Drawing}

To assess the reproducibility of the touchscreen, i.e. the ability of the participant to reproduce the figure that the model had drawn, we calculated a shape error from the difference in area between a convex hull describing the shape the model drew and a convex hull describing the shape the participant drew using the touchscreen in the scanner.

\section{RESULTS}

The results of the image quality analysis are given in Table 1. There is no apparent systematic influence of the touchscreen on any of the image quality assessments performed with the participant. As expected the percentage fluctuation in the phantom is considerably lower than the participant due to the absence of normal physiological variations induced by heart rate and breathing.

Table 1. Image quality analysis of the touchscreen

\begin{tabular}{llll}
\hline & \% Fluctuation & Drift & SFNR \\
\hline Participant drawing with touchscreen & .48 & .26 & 209.0 \\
Participant with passive touchscreen & .41 & .40 & 241.8 \\
Participant only & .47 & .36 & 212.1 \\
Phantom only & .18 & .46 & 322.8 \\
\hline
\end{tabular}


Fig. 2 shows a typical figure drawn in response to the visual Draw cue in the task. It is clear that there are no obvious influences on the reproduction of the figure induced by the time varying gradients applied during the echo-planar sequence or from the transmitted radiofrequency irradiation.
Fig. 3 shows a representative shape, as drawn by the model (panel B) and by the participant (panel A), described by a convex hull and the difference between them highlighted in green in panel $\mathrm{C}$. The normalised difference in area between the model and participant gives a measure of drawing error.

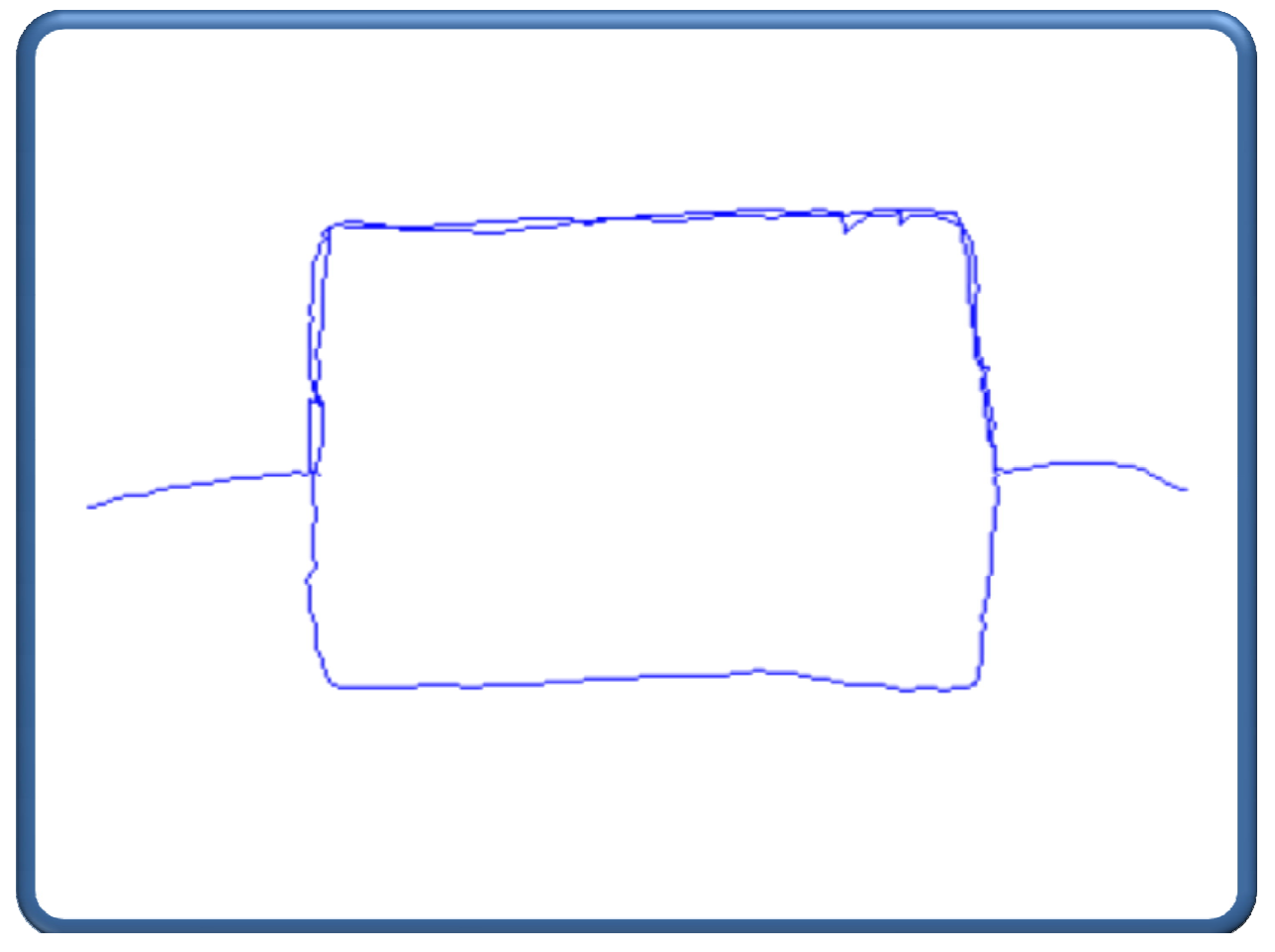

Fig. 2. Typical figure drawn by a participant in response to a video cue demonstrating little or no interference induced by the scanner

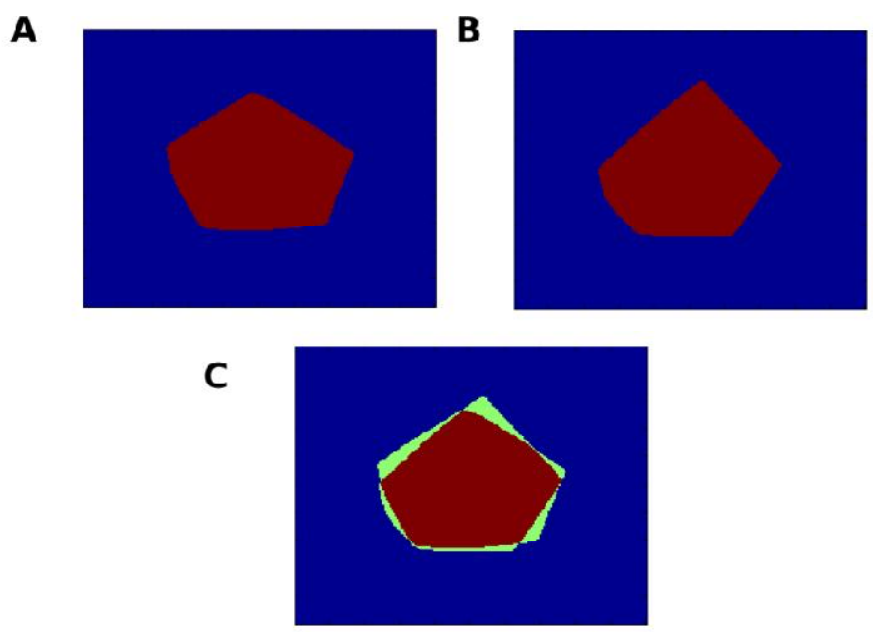

Fig. 3. Typical figure drawn by the participant $(A)$ in response to a video cue by the model (B) and the difference between them depicted by the green colouring (C) 
Mean shape error for the 18 participants was $72.33(\mathrm{SD}=16.001$, median $=67.39$; range $=$ 54.78-119.27). One person's score was larger than two standard deviations from the mean and therefore considered an outlier (error 119.27), while another participant only completed one run of the task. The error scores for the remaining 16 participants $($ mean $=69.88 ; \mathrm{SD}=11.528$; median $=67.39 ;$ range $=54.78-94.33$ ) between runs were compared using paired samples t-tests. No significant differences were found.

Group activation during Draw compared with Observe showed activation in bilateral cerebellum, insula, left supramarginal, postcentral and precentral gyri (FWE-corr. p<.05), Fig. 4.

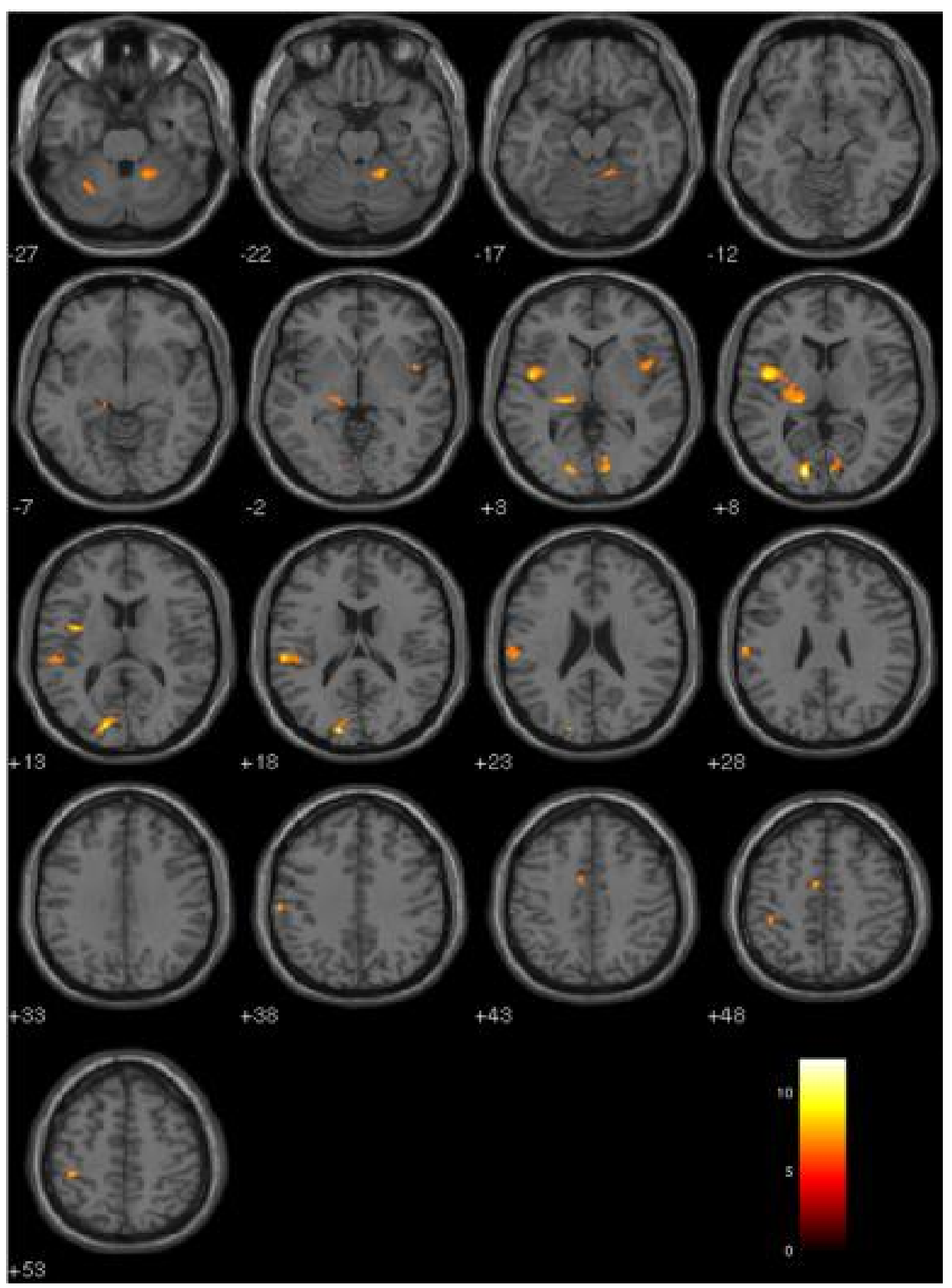

Fig. 4. Areas that showed an increase in BOLD signal in response to the Draw condition compared to the Observe condition ( $p<.05$, FWE-corr) 


\section{DISCUSSION}

This study sought to prove that a readily commercially available touchscreen could be used to investigate the neural correlates of kinematic actions in an fMRI environment. Our results show that there is no interference from the modified touchscreen on the fMRI scans, that there is no significant interference from the MRIscanner on the output of the touchscreen, and that kinematic actions performed in the scanner reveal activation in areas previously associated with more simple movements. These results have been recently corroborated by Longcamp et al. [12], who also found that using an adapted touchscreen did not affect the quality of their $\mathrm{fMRI}$ scans. The activated brain areas in Draw compared to Observe are in line with e.g. Braadbaart et al. [7] and Hanakawa, Dimyan \& Hallett [13]. Unlike the findings from Gold et al. [10], participants did not improve between runs. This might have been due to a small practice task implemented before scanning providing all the learning participants needed, or due to the touchscreen being a more valid capture of one's comparative kinematic ability rather than learning effects. More research would be needed before anything conclusive can be said about this finding.

One limitation, common to all similar systems, is that the participant is required to observe the results of their drawing efforts on a screen placed at the end of the scanner bore rather than directly as they would sitting at a desk. This may mean that inter individual differences in drawing ability, as evidenced by time dependent kinematic measures, may have an impact on the ability of the study to detect learning effects. Another limitation is that the objects that the participants were required to draw were simple (squares, circles and triangles) and it may be that we reached a ceiling effect quickly during the learning phase.

\section{CONCLUSION}

In conclusion, we have implemented a robust, simple and effective resistive touchscreen that can be used to measure the difference in drawing ability between individuals and could therefore be employed to studying the drawing or imitative abilities in clinical populations such as autism spectrum disorder or cerebral palsy.

\section{ACKNOWLEDGMENTS}

This study was funded by the Northwood Trust and the Aberdeen Biomedical Imaging Centre, University of Aberdeen. GDW is part of the SINASPE collaboration (Scottish Imaging Network - A Platform for Scientific Excellence www.SINAPSE.ac.uk). The authors thank Baljit Jagpal, Nichola Crouch, Beverly Maclennan and Katrina Klaasen for their help with running the experiment and Dawn Younie and Teresa Morris for their help with recruitment and scheduling. We also thank the participants for their generous participation.

\section{COMPETING INTERESTS}

Authors have declared that no competing interests exist.

\section{REFERENCES}

1. Culmer PR, Levesley MC, Mon-Williams $M$, Williams JHG. A new tool for assessing human movement: The kinematic assessment tool. J. Neurosci. Methods. 2009;184(1):184-92.

2. Williams JHG, Casey JM, Braadbaart L, Culmer PR, Mon-Williams M. Kinematic measures of imitation fidelity in primary school children. Journal of Cognition and Development. 2014;3(2):345-362.

3. Fadiga L, Caselli L, Craighero L, Gesierich $B$, Oliynyk A, Tia B, Viaro R. Activity in ventral premotor cortex is modulated by vision of own hand in action. Peer J. 2013; 1:e88.

4. Vogt $S$, Buccino $G$, Wohlschläger $A M$, Canessa N, Shah NJ, Zilles K, Fink GR. Prefrontal involvement in imitation learning of hand actions: Effects of practice and expertise. Neuroimage. 2007;37(4):13711383.

DOI: 10.1016/j.neuroimage.2007.07.005

5. Avikainen S, Forss N, Hari R. Modulated activation of the human SI and SIl cortices during observation of hand actions. Neuroimage. 2002;15(3):640-646.

DOI: 10.1006/nimg.2001.1029

6. McAleer P, Pollick FE, Love SA, Crabbe F, Zacks JM. The role of kinematics in cortical regions for continuous human motion perception. Cognitive, Affective, \& Behavioral Neuroscience. 2014;14(1):307318. 
7. Braadbaart L, Waiter GD, Williams JHG. Neural correlates of individual differences in manual imitation fidelity Frontiers in Integrative Neuroscience; 2012.

8. Reitz F, Richards $T, W u ~ K$, Boord $P$, Askren $\mathrm{M}$, Lewis $\mathrm{T}$, Berninger $\mathrm{V}$. A lowcost, computer-interfaced drawing pad for FMRI studies of dysgraphia and dyslexia. Sensors (Basel, Switzerland). 2013;13(4): 5099-108.

9. Tam F, Churchill NW, Strother SC, Graham SJ. A new tablet for writing and drawing during functional MRI Hum. Brain Mapp. 2011;32(2):240-8.

10. Gold BJ, Pomplun M, Rice NJ, Sekuler R. A new way to quantify the fidelity of imitation: Preliminary results with gesture sequences. Experimental Brain Research. 2008;187(1):139-152.
11. Friedman L, Glover GH. Report on a multicenter fMRI quality assurance protocol. Journal of Magnetic Resonance Imaging. 2006;23(6):827-39.

12. Longcamp $M$, Lagarrigue $A$, Nazarian $B$, Roth $\mathrm{M}$, Anton J, Alario F, Velay J. Functional specificity in the motor system: Evidence from coupled fMRI and kinematic recordings during letter and digit writing. Human Brain Mapping. 2014;35(12):60776087.

13. Hanakawa T, Dimyan MA, Hallett M. Motor planning, imagery, and execution in the distributed motor network: A time-course study with functional MRI. Cerebral Cortex (New York, N.Y.: 1991). 2008;18(12):27752788. DOI: $10.1093 /$ cercor/bhn036.

(c) 2015 Braadbaart et al.; This is an Open Access article distributed under the terms of the Creative Commons Attribution License (http://creativecommons.org/licenses/by/4.0), which permits unrestricted use, distribution, and reproduction in any medium, provided the original work is properly cited.

Peer-review history:

The peer review history for this paper can be accessed here: http://www.sciencedomain.org/review-history.php?iid=1139\&id=5\&aid=9422 